\title{
Reduced Temporal Activation During a Verbal Fluency Task is Associated with Poor Motor Speed in Patients with Major Depressive Disorder
}

\author{
Tomohiko Kiriyama $^{1 凶}$, Rumi Tanemura', Yoshihiro Nakamura², \\ Chiaki Takemoto ${ }^{2}$, Mariko Hashimoto ${ }^{2}$, and Hirohiko Utsumi ${ }^{2}$ \\ ${ }^{1}$ Department of Rehabilitation Science, Graduate School of Health Sciences, Kobe University, Kobe, Japan \\ 2Department of Psychiatry, Arima Hospital, Nishinomiya, Japan
}

\begin{abstract}
Objective Substantial research has revealed cognitive function impairments in patients with major depressive disorder (MDD). However, the relationship between MDD cognitive function impairment and brain activity is yet to be elucidated. This study aimed to reveal this relationship using near-infrared spectroscopy (NIRS) to extensively measure frontotemporal cortex function.

Methods We recruited 18 inpatients with MDD and 22 healthy controls. Regional oxygenated hemoglobin changes (oxy-Hb) were measured during a verbal fluency task and its relationship to cognitive function was assessed. Cognitive function was assessed using the Japanese version of the Brief Assessment of Cognition in Schizophrenia.

Results Compared to healthy controls, patients with MDD displayed poorer motor speed, attention and speed of information processing, and executive function. In the bilateral prefrontal and temporal surface regions, regional oxy-Hb changes were significantly lower in patients with MDD than in healthy individuals. Moreover, we observed a correlation between reduced activation in the left temporal region and poor motor speed in patients with MDD.

Conclusion We suggest that reduced activation in the left temporal region in patients with MDD could be a biomarker of poor motor speed. Additionally, NIRS may be useful as a noninvasive, clinical measurement tool for assessing motor speed in these patients.
\end{abstract}

Psychiatry Investig 2020;17(8):804-813

Key Words Major depressive disorder, Near-infrared spectroscopy, Temporal cortex, Motor speed, Cognitive function.

\section{INTRODUCTION}

Major depressive disorder (MDD), a common mental disorder worldwide, is characterized by persistent depression and loss of interest. In developed countries, its lifetime prevalence is around $15 \%{ }^{1,2} \mathrm{MDD}$ is associated not only with poor productivity but also with decreased quality of life and increased mortality. ${ }^{3}$ Deficits include cognitive, motor, perception, and communication impairments. ${ }^{4}$ Several recent reviews have reported impairments in cognitive function, including attention

Received: February 4, 2020 Revised: May 4, 2020

Accepted: May 25, 2020

$\triangle$ Correspondence: Tomohiko Kiriyama, MA

Department of Rehabilitation Science, Graduate School of Health Sciences, Kobe University, 7-10-2 Tomogaoka, Suma-ku, Kobe, Hyogo 654-0142, Japan Tel: +81-78-792-2555, Fax: +81-78-796-4509

E-mail: tomohiko_kiriyama@yahoo.co.jp

(c) This is an Open Access article distributed under the terms of the Creative Commons Attribution Non-Commercial License (https://creativecommons.org/licenses/by$\mathrm{nc} / 4.0$ ) which permits unrestricted non-commercial use, distribution, and reproduction in any medium, provided the original work is properly cited. and executive function. ${ }^{5-9}$ These cognitive impairments are related to social function, psychiatric symptoms, and prognosis. For example, with regard to neurocognitive function, impairments in visual memory, perceptual reasoning, and motor skills have been found to predict functional outcome six months later ${ }^{10}$ while impairments in executive function have a critical influence on daily life activities ${ }^{11}$ and are clearly related to suicidal ideation. ${ }^{12}$ However, the exact changes in brain functionality that cause these impairments in cognitive function in MDD remain to be elucidated.

Recent research efforts have been actively invested in elucidating the biological basis of mental illnesses. For this aim in particular, novel diagnostic imaging techniques and refined analytic methods have been developed. In MDD research, abnormalities in brain morphology and function of the cerebral neocortex have been reported, particularly in the frontotemporal cortex. ${ }^{13-15}$ Additionally, recent neurological advances have furthered understanding of the functional dif- 
ferentiation of the frontotemporal cortex, while gradually revealing the mapping of cognitive functions. ${ }^{16-19}$

Alongside functional neuroimaging techniques such as single photon emission computerized tomography (SPECT), positron emission tomography (PET), and functional magnetic resonance imaging (fMRI), near-infrared spectroscopy (NIRS) has revealed itself to be a novel, useful technique in the field of neuropsychiatric disorders for measuring brain function..$^{20-24}$ NIRS exploits the fact that near-infrared light (range, 700-1,000 nm) is partially absorbed by hemoglobin, thus allowing for the detection of intratissue hemoglobin concentration changes and the examination of intratissue blood volume and oxygen metabolism. NIRS data primarily reflects intracapillary hemoglobin and enables measurement of its oxygenated (oxy- $\mathrm{Hb})$ and deoxygenated (deoxy- $\mathrm{Hb}$ ) forms, as well as its total amount. Oxy-Hb is commonly used as the most sensitive parameter. By measuring hemoglobin concentration changes between a baseline period and a period of relative activation, NIRS allows for the examination of brain activation. Compared to other functional neuroimaging techniques, NIRS can simply and noninvasively measure spatiotemporal changes in brain function while the subject remains in a natural posture. ${ }^{25,26}$

Several studies have used NIRS to study MDD and have reported an overall lower activation reactivity during verbal fluency tasks (VFT) in bilateral prefrontal and temporal surface regions in patients with MDD compared to healthy controls, suggesting that reduced activation in these regions is a biomarker of MDD. ${ }^{27-32}$ Further, many studies have used NIRS with VFT as the activation task and have reported that functional changes in frontotemporal cortex activation correlates with MDD clinical characteristics. One such study, for example, showed evidence of reduced prefrontal activation even when clinical symptoms were in remission. ${ }^{33}$ Another reported reduced right temporal cortex activation in patients with severe negative automatic thoughts, ${ }^{34}$ while another revealed lower frontotemporal activation in patients with MDD with melancholic features than in those without melancholic features. ${ }^{35}$ The same group also reported decreased left precentral gyrus activation in suicide attempters compared to nonattempters. ${ }^{36}$ Nonetheless, several aspects of the relationship between the various clinical features of MDD, including impairment in cognitive function and the functional differentiation of frontotemporal cortex activation, remain to be elucidated. Considering that improvement in cognitive function is extremely important in treating patients with MDD, investigating this relationship will contribute to the creation of objective test methods based on biological indicators of cognitive impairment, as well as to the development of effective intervention methods for MDD.
The aim of this study was to shed light on the relationship between functional differentiation of the frontotemporal cortex and cognitive functions. To this end, we extensively examined frontotemporal cortical function in hospitalized patients with MDD using multi-channel NIRS. We hypothesize that functional activity of the frontotemporal cortex in patients with MDD is correlated to specific cognitive functions and that this relationship is discernable by NIRS.

\section{METHODS}

\section{Participants}

Participants included 18 inpatients with MDD (MDD group) and 22 healthy individuals (control group) who were matched for age, sex, years of education, and estimated intelligence quotient (IQ). All participants had an estimated IQ of 85 or above and were native speakers of Japanese. Participants in the MDD group included individuals who were inpatients at the Arima Hospital, who, during their hospitalization, were diagnosed with MDD by an experienced psychiatrist according to the guidelines of the Diagnostic and Statistical Manual of Mental Disorders 5. ${ }^{37}$ Participants were excluded if they displayed any of the following: MDD with psychotic features, history of head trauma with loss of consciousness, current or past neurological disease, current or past endocrine disease, history of treatment by electroconvulsive therapy, alcohol or substance abuse or dependence, diabetes, hypertension, heart disease, liver disease, cerebrovascular disease, and left-handedness. ${ }^{38}$ Eighteen patients (12 men, 6 women; mean age 44.2 \pm 8.7 ) on psychotropic medication at the time of testing fulfilled criteria for inclusion in the study. Among them, 17 were taking antidepressants, 7 were taking antipsychotics, 15 were taking anxiolytics, and 4 were taking mood stabilizers. Antipsychotics were being used to alleviate the symptoms of insomnia and to reinforce the antidepressant therapy. Among the four subjects prescribed mood stabilizers, two had been using lithium carbonate to reinforce the antidepressant therapy. The subjects also included some patients who, though diagnosed with MDD, were suspected of having bipolar disorder on admission. Therefore, although drug use was being minimized on admission, one patient was prescribed sodium valproate and another lamotrigine. The daily dosage of all antidepressants, antipsychotics, and anxiolytics was converted to the equivalent dose of imipramine, chlorpromazine, and diazepam, respectively. ${ }^{39}$ All patients were in a depressive state, scoring 10 points or above, on the 17-item Hamilton Rating Scale for Depression (HAM-D). ${ }^{40}$

We chose to use inpatients for this study since they are more likely to be able to receive active medical care than those receiving outpatient treatment. Therefore, early understanding 
Table 1. Demographic and clinical characteristics of patients with MDD and healthy controls

\begin{tabular}{|c|c|c|c|c|}
\hline & \multirow{2}{*}{$\begin{array}{l}\text { Patients with } \\
\text { MDD }(\mathrm{N}=18)\end{array}$} & \multirow{2}{*}{$\begin{array}{l}\text { Healthy controls } \\
\qquad(\mathrm{N}=22)\end{array}$} & \multicolumn{2}{|c|}{ Analysis } \\
\hline & & & Statistical & $\mathrm{p}$ value \\
\hline \multicolumn{5}{|l|}{ Demographic characteristics } \\
\hline Age (years) & $44.2 \pm 8.9$ & $42.0 \pm 11.2$ & $\mathrm{t}_{(\mathrm{df}=38)}=0.665$ & 0.510 \\
\hline Sex (female/male) & $6 / 12$ & $11 / 11$ & $\chi^{2}=1.13$ & 0.289 \\
\hline Education (years) & $15.0 \pm 2.5$ & $15.2 \pm 1.5$ & $\mathrm{U}=195.0$ & 0.929 \\
\hline Estimated IQ & $108.1 \pm 7.9$ & $106.3 \pm 6.8$ & $\mathrm{t}_{(\mathrm{df}=38)}=0.773$ & 0.444 \\
\hline Duration of illness (months) & $70.3 \pm 98.6$ & & & \\
\hline Depressive episodes (number) & $1.7 \pm 1.3$ & & & \\
\hline Duration of current episode (weeks) & $56.1 \pm 58.7$ & & & \\
\hline \multicolumn{5}{|l|}{ Clinical variables } \\
\hline HAM-D & $15.8 \pm 4.9$ & $1.7 \pm 1.4$ & $\mathrm{U}=0.000$ & $<0.001^{*}$ \\
\hline Task performance & $15.9 \pm 5.6$ & $15.4 \pm 4.6$ & $\mathrm{U}=195.0$ & 0.935 \\
\hline \multicolumn{5}{|l|}{ BACS-J } \\
\hline Verbal memory & $40.1 \pm 10.6$ & $44.3 \pm 7.5$ & $\mathrm{t}_{(\mathrm{df}=29.71)}=-1.436$ & 0.162 \\
\hline Working memory & $21.1 \pm 3.5$ & $21.3 \pm 4.0$ & $\mathrm{U}=190.5$ & 0.837 \\
\hline Motor Speed & $79.9 \pm 15.4$ & $88.9 \pm 7.6$ & $\mathrm{U}=123.5$ & $0.042^{*}$ \\
\hline Verbal fluency & $47.8 \pm 12.4$ & $46.1 \pm 10.0$ & $\mathrm{t}_{(\mathrm{df}=38)}=0.480$ & 0.634 \\
\hline Attention and speed of information processing & $58.9 \pm 8.3$ & $66.4 \pm 7.1$ & $\mathrm{t}_{(\mathrm{df}=38)}=-3.060$ & $0.004^{*}$ \\
\hline Executive function & $17.3 \pm 3.1$ & $19.0 \pm 1.8$ & $\mathrm{U}=127.0$ & $0.049^{*}$ \\
\hline Imipramine equivalent dose $(\mathrm{mg})^{\dagger}$ & $165.4 \pm 129.9$ & & & \\
\hline Chlorpromazine equivalent dose $(\mathrm{mg})^{\ddagger}$ & $23.3 \pm 35.9$ & & & \\
\hline Diazepam equivalent dose (mg)§ & $15.4 \pm 25.1$ & & & \\
\hline
\end{tabular}

Data are presented as mean \pm standard deviation. ${ }^{*}$ Significant $p$ values, ${ }^{\dagger}$ antidepressant dosages were evaluated using the imipramine equivalent dosage, ‡antipsychotic dosages were evaluated using the chlorpromazine equivalent dosage, \$anxiolytic dosages were evaluated using the diazepam equivalent dosage. MDD: major depressive disorder, IQ: intelligence quotient, HAM-D: 17-item Hamilton Rating Scale for Depression, BACS-J: Japanese version of the Brief Assessment of Cognition in Schizophrenia

of the condition on hospitalization allows for the administration of more appropriate treatment on a priority basis.

Healthy individuals who were appropriately age- and sexmatched to the MDD group participated as controls in the present study. Inclusion criteria were the same as those for the MDD group, but also included no current or past history of psychiatric consultation, no history of psychiatric illness in second-degree relatives, and no psychotropic medication taken at the time of testing. Twenty-two healthy controls (11 men, 11 women; mean age $42.0 \pm 10.9$ ) fulfilled these criteria.

Demographic and clinical characteristics of all participants are summarized in Table 1. As evaluated by chi-squared tests, Student's t-tests, and Mann-Whitney U tests, we did not observe any significant differences between groups for sex, age, or years of education. All tests, including NIRS, were conducted on the same day.

This study was approved by the ethical review board of the Arima Hospital (IRB approval number: 2015-4) and the Kobe University Graduate School of Health Sciences (IRB approval number: 404-1). All participants received a full briefing of the goal and details of the study prior to participation. All participants provided written consent. The research was conducted in compliance with guidelines set forth by the World Medical Association Declaration of Helsinki.

\section{Clinical assessment}

Depression symptoms were assessed using the 17-item HAM-D. ${ }^{40}$ Estimated IQ was calculated using the Japanese version of the National Adult Reading Test. ${ }^{41}$

\section{Cognitive assessment}

Cognitive function was evaluated using the Japanese version of the Brief Assessment of Cognition in Schizophrenia (BACS-J). ${ }^{42,43}$ Though the BACS-J is a test of neurocognition for schizophrenia, it has also been used for MDD. ${ }^{44-46}$ It is composed of six subscales: verbal memory, working memory, motor speed, verbal fluency, attention and speed of information processing, and executive function. A higher score 
reflects higher functioning across all scales. We used raw scores for all statistical analyses performed in this study.

\section{VFT and NIRS measurements}

Hemoglobin concentration changes were measured during VFT trials while participants sat in a chair with their eyes open. The VFT was performed according to the protocol laid out by Takizawa et al. ${ }^{28}$ The protocol used was similar to that used in the neuroimaging-aided differential diagnosis of depressive states. The imaging time was $30 \mathrm{~s}$ for acquiring the pre-task baseline, $60 \mathrm{~s}$ for the VFT, and $70 \mathrm{~s}$ for acquiring the post-task baseline. In both baseline conditions (pre- and posttask), the five vowels of the Japanese language "a, i, u, e, o" were repeated. The VFT condition was divided into three 20-s periods; in the first, second, and third period, the subject was asked to state as many words as possible beginning with the sounds "a," "ki," and "ha," respectively. Task performance was calculated according to the total number of words answered correctly (Figure 1).

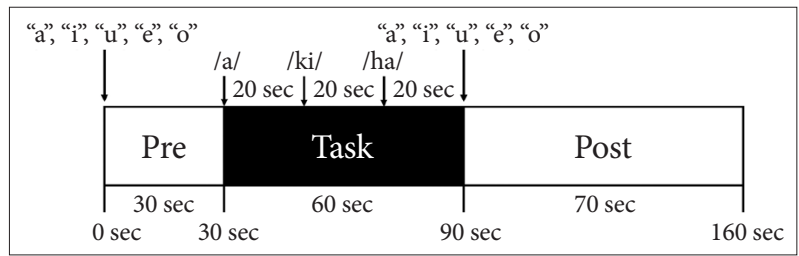

Figure 1. Design of the verbal fluency task.
Relative oxy-Hb/deoxy-Hb changes were measured based on the modified Beer-Lambert $\mathrm{Law}^{47}$ with a 52-channel NIRS device (ETG-4000 Optical Topography System; Hitachi Medical Co., Tokyo, Japan) using two wavelengths of near-infrared light, 695 and $830 \mathrm{~nm}$. This method allows for the calculation of signals reflecting $\mathrm{Hb}$ concentration changes and their conversion to arbitrary units ( $\mathrm{mM} \cdot \mathrm{mm})$. The device beams nearinfrared light onto the cranium and measures the relative change in the amount of light that is reflected back to the scalp after diffusion through cranial tissues. The distance between each pair of source-detector probes was set at $3 \mathrm{~cm}$, and the area measured between the pair of probes was defined as a "channel." Since the NIRS device measures the mean amount of blood within $2-3 \mathrm{~cm}$ from the scalp, it can measure changes in cerebral cortical activity. ${ }^{48,49}$ NIRS probes were fixed in place with $3 \times 11$ thermoplastic shells. The bottom row of measurement probes was placed above $\mathrm{Fp} 1$ and $\mathrm{Fp} 2$, according to the international 10-20 method (Figure 2A). We utilized 33 probes which consisted of 17 light emitters and 16 detectors. Therefore, the probe consisted of 52 channels (Figure 2B). The spatial information of the respective channels was estimated using data from the Functional Brain Science Laboratory at the Jichi Medical University, Japan. ${ }^{50,51}$ We hypothesized that NIRS channels can measure hemoglobin concentration in areas corresponding to the bilateral frontal, temporal, and parietal cortices according to LONI Probabilistic Brain Atlas $(\text { LPBA40) })^{52}$ (Figure 2C-E).
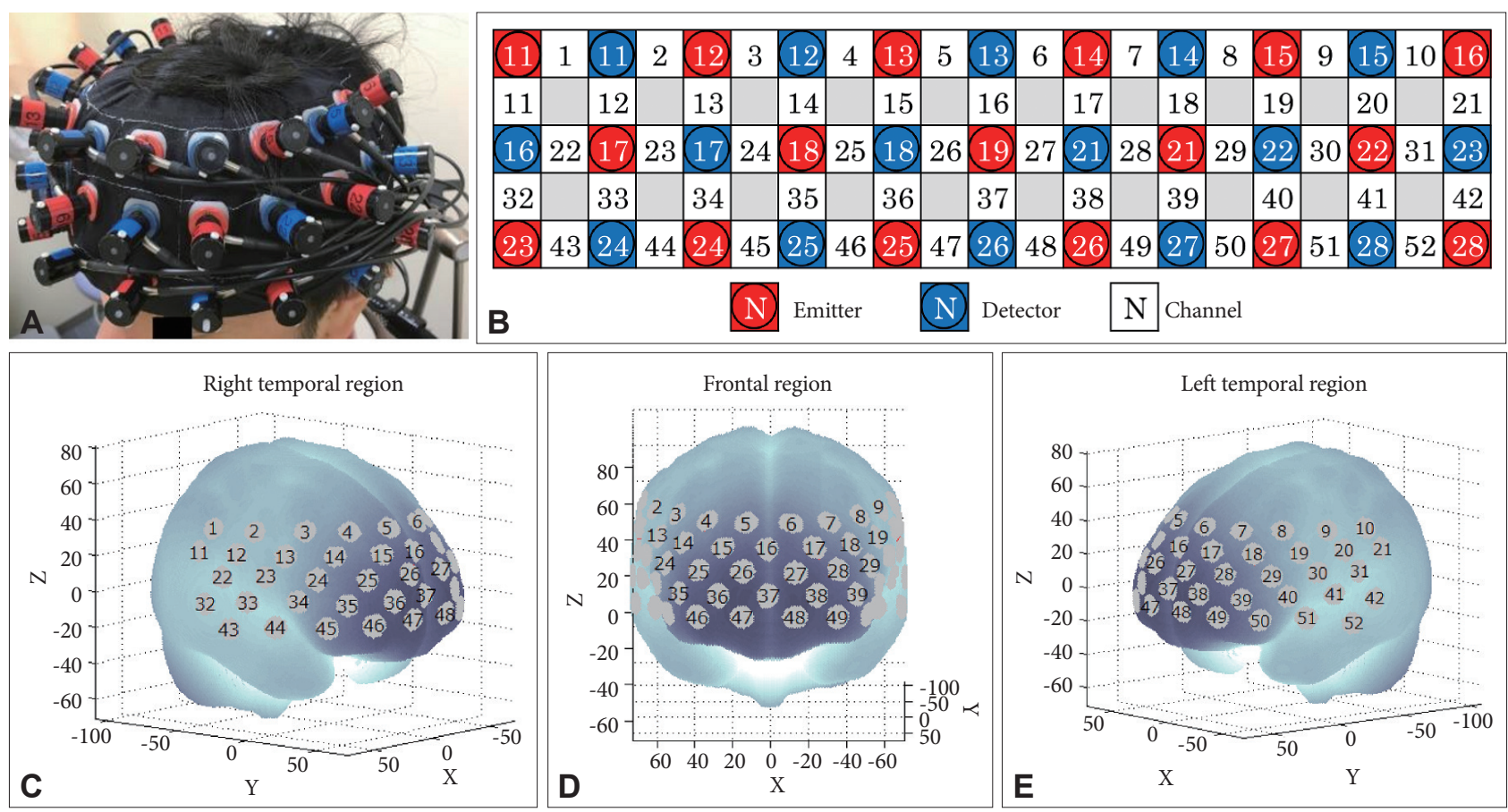

Figure 2. Measurement points for the 52 NIRS channels. A: Probes with $3 \times 11$ thermoplastic shells were placed on the scalp of the subject over the bilateral frontotemporal regions. B: Placement of the 17 emitters and 16 detectors. The 52 measurement areas are labeled ch1ch52, from the right posterior to the left anterior region. C-E: Anatomical positions corresponding to each channel. 
The temporal resolution of the NIRS device was set to $0.1 \mathrm{~s}$. The obtained data were analyzed using the 'integral mode', using a 10-s pre-task baseline interval immediately before the VFT, and a 5-s post-task baseline interval, beginning $50 \mathrm{~s}$ after the end of the VFT. We created a first-order correction baseline using the least-squares method, based on these two baseline intervals, and calculated the amount of change from baseline. To eliminate high frequency noise, such as heartbeats or small body movements, we set the moving average method to $5 \mathrm{~s}$. Using previously adopted computer algorithms, ${ }^{53}$ we excluded channel data with excessive artifacts, which explains the different number of participants for each channel.

Simultaneous NIRS and fMRI studies have strongly correlated cerebral blood flow to oxy-Hb rather than to deoxy$\mathrm{Hb}^{54}$ Therefore, we focused on oxy-Hb data.

\section{Statistical analysis}

We confirmed the normal distribution of demographic data (age, education, estimated IQ), HAM-D score, cognitive function (scores from the six subscales for BACS-J), and task performance using the Shapiro-Wilk test, after which we performed a chi-squared test, Student's t-test, or Mann-Whitney $\mathrm{U}$ test, as appropriate, to assess differences between the MDD and control groups.

A student's t-test was performed to compare differences in mean VFT-induced oxy-Hb changes for each channel between the two groups. Since there were 52 channels to be analyzed, ttests were performed 52 times. The use of many channels in the analysis incurred a high likelihood of introducing a type II error should a Bonferroni correction be used for multiple comparisons. Therefore, a false discovery rate (FDR)-based procedure was adopted to correct for multiple comparisons. To ensure that the false positive rate did not exceed $5 \%$ on average, we set a q value establishing a maximum FDR of $0.05 .^{55}$ To assess correlations between mean oxy- $\mathrm{Hb}$ changes and demographic and clinical variables (age, years of education, estimated IQ, HAM-D score, illness duration, number of depressive episodes, duration of depressive episodes, task performance, imipramine dose, chlorpromazine dose, and diazepam dose) in the MDD group, we conducted Pearson's or Spearman's correlation analysis, as appropriate, and applied an FDR correction.

Additionally, to assess correlation between mean oxy- $\mathrm{Hb}$ changes and cognitive function (scores from the six subscales for BACS-J) in the MDD group, we conducted a Pearson's or Spearman's correlation analysis, as appropriate, and applied an FDR correction.

Finally, a correlation analysis was carried out with a control group to assess whether the correlation detected in the aforementioned correlation analysis was reproducible. Statistical analyses were conducted using the Statistical Package for the
Social Sciences (SPSS) version 24.0 (IBM Corp., Armonk, NY, USA) in Windows.

\section{RESULTS}

\section{Demographic data, depression severity, and task performance}

Demographic and clinical data are shown in Table 1. The HAM-D score was significantly higher in the MDD than in the control group $(\mathrm{U}=0.000, \mathrm{p}<0.001)$. We did not detect a significant difference in the number of generated words in the VFT $(\mathrm{U}=195.0, \mathrm{p}=0.935)$ (Table 1).

\section{Cognitive functions}

We found significant differences between the control and MDD groups, with the MDD group performing more poorly on the following subscales scores of the BACS-J: motor speed $(\mathrm{U}=123.5, \mathrm{p}=0.042)$, attention and speed of information processing $(\mathrm{t}=-3.060, \mathrm{p}=0.004)$, and executive function $(\mathrm{U}=127.0$, $\mathrm{p}=0.049)$ (Table 1$)$.

\section{Mean oxy-Hb changes during the VFT}

Mean VFT-induced oxy-Hb changes were significantly lower in patients with MDD than in healthy individuals for 15 channels located in the bilateral prefrontal and temporal surface regions (ch15, ch22, ch26-27, ch30, ch32-33, ch38, ch40-42, ch44, ch48, ch51-52; FDR-corrected $\mathrm{p}=0.0006$ to 0.0137 ) (Figure 3 ).

\section{Correlation analyses}

In the MDD group, the number of depressive episodes was significantly negatively correlated with the mean VFT-induced oxy-Hb changes in two channels covering the left superior temporal gyrus (ch31, 42), left supramarginal gyrus (ch31), left angular gyrus (ch31), and left middle temporal gyrus (ch42) (ch31, ch42; rho $=-0.745$ to -0.603 , FDR-corrected $\mathrm{p}=0.0010$ to 0.0014). After multiple comparisons correction with FDR, there was no significant correlation between the mean oxy- $\mathrm{Hb}$ changes and other demographic or clinical variables (age, years of education, estimated IQ, HAM-D score, illness duration, duration of depressive episodes, task performance, imipramine dose, chlorpromazine dose, and diazepam dose) (Supplementary Table 1 in the online-only Data Supplement).

In the MDD group, the BACS-J motor speed score was significantly positively correlated with mean oxy-Hb changes in one channel covering the left superior temporal gyrus and the left middle temporal gyrus (ch52; rho=0.904, FDR-corrected $\mathrm{p}<0.0001$ ) (Figure 4). However, no significant correlation was found between the other five BACS-J subscale scores (verbal memory, working memory, verbal fluency, attention and speed of information processing, and executive 
function) and mean oxy-Hb changes after multiple comparisons correction with FDR (Supplementary Table 2 in the online-only Data Supplement).
For healthy participants, we failed to find any significant correlation between the BACS-J motor speed and the mean oxy$\mathrm{Hb}$ changes after multiple comparisons correction with FDR
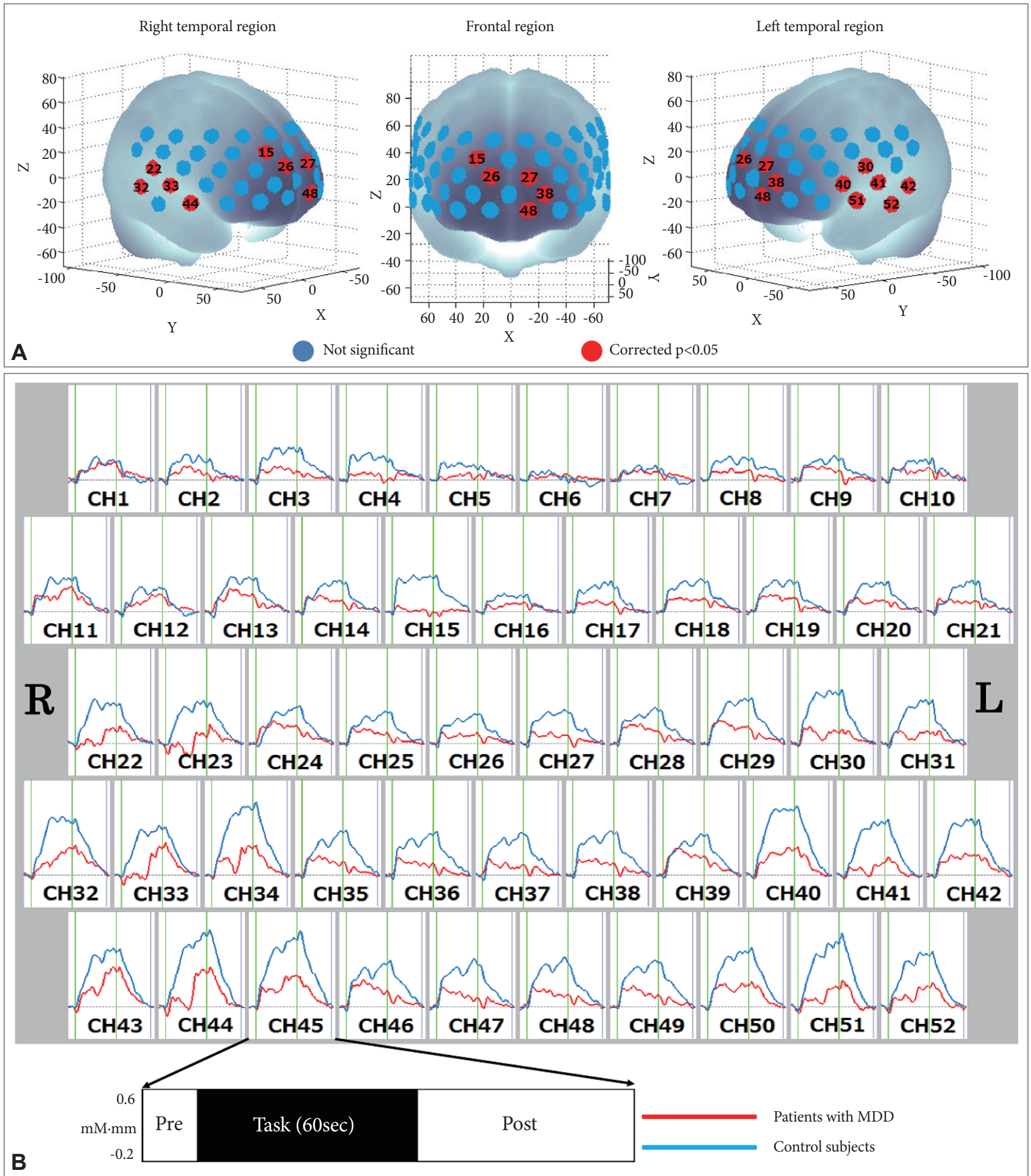

Figure 3. Comparison of mean oxy-Hb changes during the VFT. A: Brain regions shown in red correspond to NIRS channels with significantly lower levels of mean oxy-Hb changes in patients with MDD than in healthy controls (FDR-corrected $\mathrm{p}<0.05$ ), and those shown in blue are not significant. B: Grand-averaged waveforms of the mean oxy-Hb changes in patients with MDD and healthy controls. MDD: major depressive disorder, oxy-Hb: oxygenated hemoglobin, VFT: verbal fluency task, NIRS: near-infrared spectroscopy, FDR: false discovery rate. 

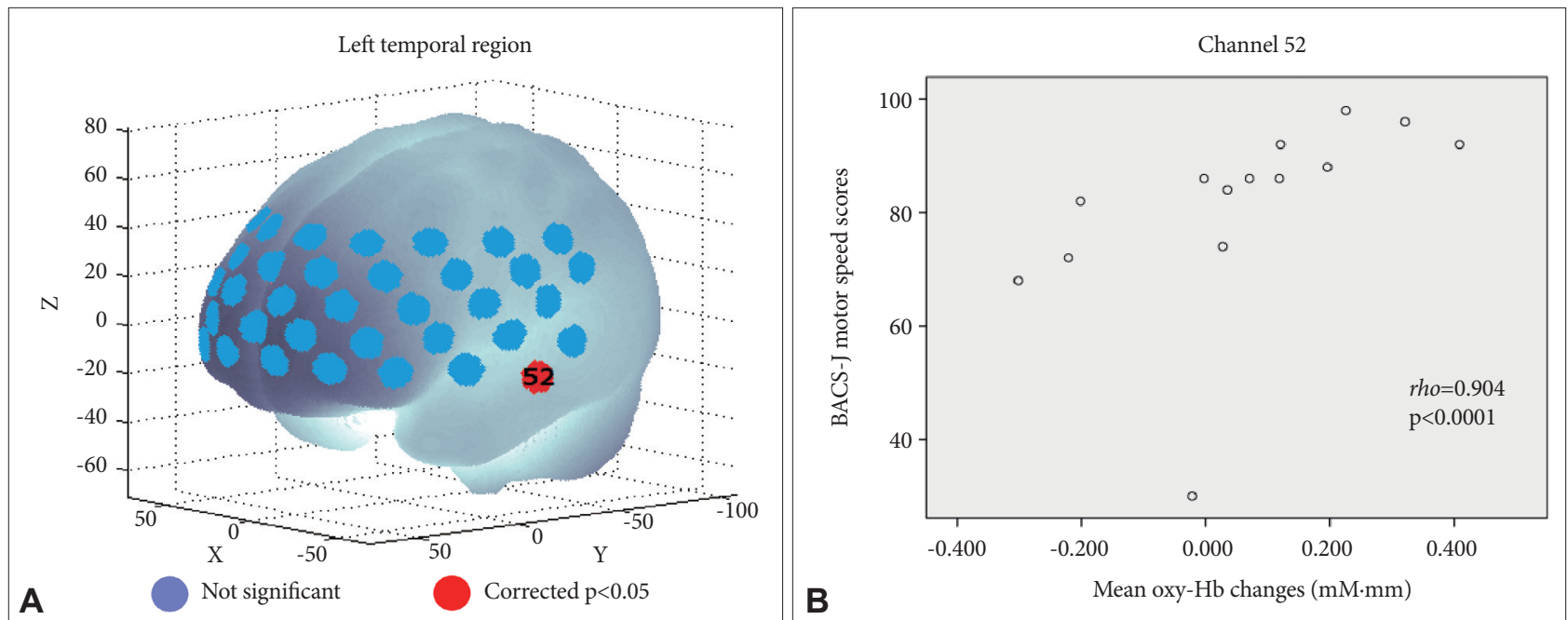

Figure 4. Relationship between mean oxy-Hb changes and motor speed scores in patients with MDD. A: Significant correlation is shown as a red-colored channel (FDR-corrected $p<0.05$ ), and non-significant correlations are shown as blue-colored channels. B: Scatter diagram showing the relationship between motor speed scores and mean oxy-Hb changes in ch52. MDD: major depressive disorder, FDR: false discovery rate.

for any channel ( $r h o=-0.591$ to $0.253, \mathrm{p}=0.0047$ to 1.0000$)$.

\section{DISCUSSION}

\section{Cognitive function}

This study demonstrated that patients with MDD perform more poorly in motor speed, attention and speed of information processing, and executive function than healthy controls matched for age, sex, and years of education. These three neurocognitive dysfunctions were also observed in a previous study using BACS-J. ${ }^{46}$

\section{Mean oxy-Hb changes during the VFT}

Although we did not detect differences in the number of generated words in the VFT between the MDD and control groups, the mean VFT-induced oxy-Hb changes in the entire bilateral prefrontal and temporal surface regions were lower in the MDD than the control group. This result is consistent with previous reports ${ }^{27,29-32}$ and suggests that reduced mean oxy- $\mathrm{Hb}$ changes in the bilateral prefrontal and temporal surface regions may constitute a biomarker for MDD.

\section{Correlation analyses}

We found that the number of depressive episodes was significantly negatively correlated with the mean VFT-induced oxy-Hb changes in the left superior temporal gyrus, left supramarginal gyrus, left angular gyrus, and left middle temporal gyrus. It has been reported that the length of past depressive episodes negatively influences memory performance ${ }^{56}$; accordingly, more depressive episodes may also reduce activation of the left temporal region and subparietal district.
We found that motor speed scores positively correlated with the mean VFT-induced oxy-Hb changes in the left superior temporal gyrus and left middle temporal gyrus. These findings indicated that the more the motor speed is limited, the further these changes are reduced. In this study, a token motor task was used to measure the motor speed. In this task, the subjects are given 100 plastic tokens and instructed to place them two at a time into a container as quickly as possible. ${ }^{42}$ The temporal lobe accounts for $17 \%$ of the cerebral cortex and includes areas with auditory, olfactory, vestibular, visual, and linguistic functions. ${ }^{57}$ This is consistent with the findings of previous studies showing that the temporal area is activated during a VFT ${ }^{58}$ and is involved in visuomotor coordination. ${ }^{59}$ Additionally, in a study on MDD evaluation using SPECT, researchers found a negative correlation between psychomotor retardation severity, measured by the Unified Parkinson's disease rating scale (UPDRS), and frontal and temporal perfusion. ${ }^{60}$ Moreover, studies using NIRS have reported a negative correlation between psychomotor retardation score, measured by the HAM-D, and mean VFT-induced oxy- $\mathrm{Hb}$ changes in the frontal and bilateral temporal regions in patients with $\mathrm{MDD},{ }^{30}$ or in the frontal and left temporal regions in those with MDD with non-melancholic features. ${ }^{35}$ Our study primarily demonstrated a relationship between the left temporal region and motor speed, consistent with these previous studies. However, our study provides further significant quantitative and objective support to this relationship, since it quantitively assessed motor speed using the BACS-J. These previous studies, in contrast, had assessed psychomotor function based solely on interviews and clinical observation using the UPDRS and HAM-D.

Since we did not observe a relationship between motor 
speed and temporal region activation in healthy controls, we consider that the presence of such correlation is specific to patients with MDD. However, it remains unclear whether these results are due to abnormal neural correlates or compensatory mechanisms. Nonetheless, in MDD, the relationship between temporal region activation and motor speed likely demonstrates its essential role in maintaining motor speed. Overall, our results further the understanding of MDD pathophysiology.

\section{Limitations}

This study has a few limitations. First, we utilized multiple comparison corrections to avoid Type I errors, but due to the relatively small sample size, we cannot rule out the possibility of Type II errors. Second, although depressive symptoms primarily ranged in severity from mild to moderate, since all participants were inpatients, it remains unclear whether the results are reproducible in an outpatient cohort. Additionally, inpatients undergo a number of therapeutic changes over the course of their treatment, making it difficult to properly assess baseline cerebral metabolism. Third, not all medications were stringently regulated. Although the discriminating MDD, bipolar disorder, and schizophrenia were similar between a small group not administered with the drug and that administered with the drug. ${ }^{28}$ Further evidence is needed to elucidate the effect of drugs on cerebral metabolism.

\section{Conclusions}

We extensively investigated frontotemporal cortical function in patients with MDD using multi-channel NIRS. As expected, we discovered that functional differentiation of the frontotemporal cortex correlates with cognitive function. We suggest that reductions in VFT-induced mean oxy-Hb changes in the left superior temporal gyrus and the left middle temporal gyrus may be used as a biomarker for poor motor speed in MDD. We propose that NIRS can not only assist in the differential diagnosis of MDD but can also be used for assessing poor motor speed.

\section{Supplementary Materials}

The online-only Data Supplement is available with this article at https://doi.org/10.30773/pi.2020.0045.

\section{Acknowledgments}

\section{None.}

\section{Conflicts of Interest}

The authors have no potential conflicts of interest to disclose.

\section{Author Contributions}

Conceptualization: Tomohiko Kiriyama, Rumi Tanemura. Data curation: Tomohiko Kiriyama, Yoshihiro Nakamura, Chiaki Takemoto, Mariko
Hashimoto, Hirohiko Utsumi. Formal analysis: Tomohiko Kiriyama, Rumi Tanemura. Investigation: all authors. Methodology: Tomohiko Kiriyama, Rumi Tanemura. Project administration: Tomohiko Kiriyama, Rumi Tanemura. Supervision: Rumi Tanemura. Validation: Rumi Tanemura. Visualization: Tomohiko Kiriyama, Rumi Tanemura. Writing-original draft: Tomohiko Kiriyama, Rumi Tanemura. Writing_review \& editing: all authors.

\section{ORCID iDs}

Tomohiko Kiriyama

Rumi Tanemura

Yoshihiro Nakamura

Chiaki Takemoto

Mariko Hashimoto

Hirohiko Utsumi

\begin{abstract}
https://orcid.org/0000-0003-3368-320X https://orcid.org/0000-0002-3390-9080 https://orcid.org/0000-0001-7725-2351 https://orcid.org/0000-0001-5364-7746 https://orcid.org/0000-0001-7020-7793 https://orcid.org/0000-0001-7932-1429
\end{abstract}

\section{REFERENCES}

1. Bromet E, Andrade LH, Hwang I, Sampson NA, Alonso J, de Girolamo G, et al. Cross-national epidemiology of DSM-IV major depressive episode. BMC Med 2011;9:90.

2. Kessler RC, McGonagle KA, Swartz M, Blazer DG, Nelson CB. Sex and depression in the National Comorbidity Survey. I: Lifetime prevalence, chronicity and recurrence. J Affect Disord 1993;29:85-96.

3. Doris A, Ebmeier K, Shajahan P. Depressive illness. Lancet 1999;354: 1369-1375.

4. Miller WR. Psychological deficit in depression. Psychol Bull 1975;82: 238-260.

5. Bora E, Harrison BJ, Yücel M, Pantelis C. Cognitive impairment in euthymic major depressive disorder: a meta-analysis. Psychol Med 2013; 43:2017-2026.

6. Lee RS, Hermens DF, Porter MA, Redoblado-Hodge MA. A metaanalysis of cognitive deficits in first-episode Major Depressive Disorder. J Affect Disord 2012;140:113-124.

7. Snyder HR. Major depressive disorder is associated with broad impairments on neuropsychological measures of executive function: a metaanalysis and review. Psychol Bull 2013;139:81-132.

8. Trivedi MH, Greer TL. Cognitive dysfunction in unipolar depression: implications for treatment. J Affect Disord 2014;152-154:19-27.

9. Wagner S, Doering B, Helmreich I, Lieb K, Tadić A. A meta-analysis of executive dysfunctions in unipolar major depressive disorder without psychotic symptoms and their changes during antidepressant treatment. Acta Psychiatr Scand 2012;125:281-292.

10. Jaeger J, Berns S, Uzelac S, Davis-Conway S. Neurocognitive deficits and disability in major depressive disorder. Psychiatry Res 2006;145: 39-48.

11. Kiosses DN, Alexopoulos GS. IADL functions, cognitive deficits, and severity of depression: a preliminary study. Am J Geriatr Psychiatry 2005; 13:244-249.

12. Westheide J, Quednow BB, Kuhn KU, Hoppe C, Cooper-Mahkorn D, Hawellek, et al. Executive performance of depressed suicide attempters: the role of suicidal ideation. Eur Arch Psychiatry Clin Neurosci 2008;258:414-421.

13. Drevets WC. Neuroimaging studies of mood disorders. Biol Psychiatry 2000;48:813-829.

14. Lorenzetti V, Allen NB, Fornito A, Yücel M. Structural brain abnormalities in major depressive disorder: a selective review of recent MRI studies. J Affect Disord 2009;117:1-17.

15. Rogers MA, Kasai K, Koji M, Fukuda R, Iwanami A, Nakagome K, et al. Executive and prefrontal dysfunction in unipolar depression: a review of neuropsychological and imaging evidence. Neurosci Res 2004;50:1-11.

16. Cox SR, Ferguson KJ, Royle NA, Shenkin SD, MacPherson SE, MacLullich AM, et al. A systematic review of brain frontal lobe parcellation techniques in magnetic resonance imaging. Brain Struct Funct 2014;219:1-22. 
17. Erickson LC, Rauschecker JP, Turkeltaub PE. Meta-analytic connectivity modeling of the human superior temporal sulcus. Brain Struct Funct 2017;222:267-285.

18. Squire LR, Stark CE, Clark RE. The medial temporal lobe. Annu Rev Neurosci 2004;27:279-306.

19. Szczepanski SM, Knight RT. Insights into human behavior from lesions to the prefrontal cortex. Neuron 2014;83:1002-1018.

20. Hirosawa R, Narumoto J, Sakai Y, Nishida S, Ishida T, Nakamae T, et al. Reduced dorsolateral prefrontal cortical hemodynamic response in adult obsessive-compulsive disorder as measured by near-infrared spectroscopy during the verbal fluency task. Neuropsychiatr Dis Treat 2013;9:955-962.

21. Kameyama M, Fukuda M, Yamagishi Y, Sato T, Uehara T, Ito M, et al. Frontal lobe function in bipolar disorder: a multichannel near-infrared spectroscopy study. Neuroimage 2006;29:172-184.

22. Matsuo K, Taneichi K, Matsumoto A, Ohtani T, Yamasue H, Sakano Y, et al. Hypoactivation of the prefrontal cortex during verbal fluency test in PTSD: a near-infrared spectroscopy study. Psychiatry Res 2003;124:1-10.

23. Matsuo K, Onodera Y, Hamamoto T, Muraki K, Kato N, Kato T. Hypofrontality and microvascular dysregulation in remitted late-onset depression assessed by functional near-infrared spectroscopy. Neuroimage 2005;26:234-242.

24. Suto T, Fukuda M, Ito M, Uehara T, Mikuni M. Multichannel near-infrared spectroscopy in depression and schizophrenia: cognitive brain activation study. Biol Psychiatry 2004;55:501-511.

25. Boas DA, Dale AM, Franceschini, MA. Diffuse optical imaging of brain activation: approaches to optimizing image sensitivity, resolution, and accuracy. Neuroimage 2004;23:S275-288.

26. Strangman G, Boas DA, Sutton JP. Non-invasive neuroimaging using near-infrared light. Biol Psychiatry 2002;52:679-693.

27. Tomioka H, Yamagata B, Kawasaki S, Pu S, Iwanami A, Hirano J, et al. A longitudinal functional neuroimaging study in medication-naïve depression after antidepressant treatment. PLoS One 2015;10:e0120828.

28. Takizawa R, Fukuda M, Kawasaki S, Kasai K, Mimura M, Pu S, et al. Neuroimaging-aided differential diagnosis of the depressive state. Neuroimage 2014;85:498-507.

29. Liu X, Sun G, Zhang X, Xu B, Shen C, Shi L, et al. Relationship between the prefrontal function and the severity of the emotional symptoms during a verbal fluency task in patients with major depressive disorder: a multi-channel NIRS study. Prog Neuropsychopharmacol Biol Psychiatry 2014;54:114-121.

30. Noda T, Yoshida S, Matsuda T, Okamoto N, Sakamoto K, Koseki S, et al. Frontal and right temporal activations correlate negatively with depression severity during verbal fluency task: a multi-channel near-infrared spectroscopy study. J Psychiatr Res 2012;46:905-912.

31. Pu S, Matsumura H, Yamada T, Ikezawa S, Mitani H, Adachi A, et al. Reduced frontopolar activation during verbal fluency task associated with poor social functioning in late-onset major depression: multichannel near-infrared spectroscopy study. Psychiatry Clin Neurosci 2008;62:728-737.

32. Pu S, Nakagome K, Yamada T, Yokoyama K, Matsumura H, Mitani H, et al. The relationship between the prefrontal activation during a verbal fluency task and stress-coping style in major depressive disorder: a near-infrared spectroscopy study. J Psychiatr Res 2012;46:1427-1434.

33. Ikeda E, Shiozaki K, Ikeda H, Suzuki M, Hirayasu Y. Prefrontal dysfunction in remitted depression at work reinstatement using near-infrared spectroscopy. Psychiatry Res 2013;214:254-259.

34. Koseki S, Noda T, Yokoyama S, Kunisato Y, Ito D, Suyama H, et al. The relationship between positive and negative automatic thought and activity in the prefrontal and temporal cortices: a multi-channel near-infrared spectroscopy (NIRS) study. J Affect Disord 2013;151:352-359.

35. Tsujii N, Mikawa W, Akashi H, Tsujimoto E, Adachi T, Kirime E, et al. Right temporal activation differs between melancholia and nonmelancholic depression: a multichannel near-infrared spectroscopy study. J Psychiatr Res 2014;55:1-7.
36. Tsujii N, Mikawa W, Tsujimoto E, Adachi T, Niwa A, Ono H, et al. Reduced left precentral regional responses in patients with major depressive disorder and history of suicide attempts. PLoS One 2017;12: e0175249.

37. American Psychiatric Association. Diagnostic and Statistical Manual of Mental Disorders (5th Ed). Washington, D.C.: American Psychiatric Publishing; 2013.

38. Oldfield RC. The assessment and analysis of handedness: the Edinburgh inventory. Neuropsychologia 1971;9:97-113.

39. Inada T, Inagaki A. Psychotropic dose equivalence in Japan. Psychiatry Clin Neurosci 2015;69:440-447.

40. Hamilton M. A rating scale for depression. J Neurol Neurosurg Psychiatry 1960;23:56-62.

41. Matsuoka K, Uno M, Kasai K, Koyama K, Kim Y. Estimation of premorbid IQ in individuals with Alzheimer's disease using Japanese ideographic script (Kanji) compound words: Japanese version of National Adult Reading Test. Psychiatry Clin Neurosci 2006;60:332-339.

42. Keefe RS, Goldberg TE, Harvey PD, Gold JM, Poe MP, Coughenour L. The Brief Assessment of Cognition in Schizophrenia: reliability, sensitivity, and comparison with a standard neurocognitive battery. Schizophr Res 2004;68:283-297.

43. Kaneda Y, Sumiyoshi T, Keefe R, Ishimoto Y, Numata S, Ohmori T. Brief assessment of cognition in schizophrenia: validation of the Japanese version. Psychiatry Clin Neurosci 2007;61:602-609.

44. Pu S, Setoyama S, Noda T. Association between cognitive deficits and suicidal ideation in patients with major depressive disorder. Sci Rep 2017;7:11637.

45. Hidese S, Ota M, Wakabayashi C, Noda T, Ozawa H, Okubo T, et al. Effects of chronic l-theanine administration in patients with major depressive disorder: an open-label study. Acta Neuropsychiatr 2017;29:72-79.

46. Hidese S, Ota M, Matsuo J, Ishida I, Hiraishi M, Yoshida S, et al. Association of obesity with cognitive function and brain structure in patients with major depressive disorder. J Affect Disord 2018;225:188-194.

47. Yamashita Y, Maki A, Ito Y, Watanabe E, Koizumi H. Noninvasive near-infrared topography of human brain activity using intensity modulation spectroscopy. Opt Eng 1996;35:1046-1049.

48. Toronov V, Webb A, Choi JH, Wolf M, Michalos A, Gratton E, et al. Investigation of human brain hemodynamics by simultaneous nearinfrared spectroscopy and functional magnetic resonance imaging. Med Phys 2001;28:521-527.

49. Okada E, Delpy DT. Near-infrared light propagation in an adult head model. II. Effect of superficial tissue thickness on the sensitivity of the near-infrared spectroscopy signal. Appl Opt 2003;42:2915-2922.

50. Singh AK, Okamoto M, Dan H, Jurcak V, Dan I. Spatial registration of multichannel multi-subject fNIRS data to MNI space without MRI. Neuroimage 2005;27:842-851.

51. Tsuzuki D, Jurcak V, Singh AK, Okamoto M, Watanabe E, Dan I. Virtual spatial registration of stand-alone fNIRS data to MNI space. Neuroimage 2007;34:1506-1518.

52. Shattuck DW, Mirza M, Adisetiyo V, Hojatkashani C, Salamon G, Narr $\mathrm{KL}$, et al. Construction of a 3D probabilistic atlas of human cortical structures. Neuroimage 2007;39:1064-1080.

53. Takizawa R, Kasai K, Kawakubo Y, Marumo K, Kawasaki S, Yamasue $\mathrm{H}$, et al. Reduced frontopolar activation during verbal fluency task in schizophrenia: a multi-channel near-infrared spectroscopy study. Schizophr Res 2008;99:250-262.

54. Strangman G, Culver JP, Thompson JH, Boas DA. A quantitative comparison of simultaneous BOLD fMRI and NIRS recordings during functional brain activation. Neuroimage 2002;17:719-731.

55. Singh AK, Dan I. Exploring the false discovery rate in multichannel NIRS. Neuroimage 2006;33:542-549.

56. Gorwood P1, Corruble E, Falissard B, Goodwin GM. Toxic effects of depression on brain function: impairment of delayed recall and the cumulative length of depressive disorder in a large sample of depressed outpatients. Am J Psychiatry 2008:165:731-739. 
57. Kiernan JA. Anatomy of the temporal lobe. Epilepsy Res Treat 2012; 2012:176157.

58. Pirmoradi M, Jemel B, Gallagher A, Tremblay J, D’Hondt F, Nguyen $\mathrm{DK}$, et al. Verbal memory and verbal fluency tasks used for language localization and lateralization during magnetoencephalography. Epi- lepsy Res 2016;119:1-9.

59. Tankus A, Fried I. Visuomotor coordination and motor representation by human temporal lobe neurons. J Cogn Neurosci 2012;24:600-610. 60. Mayberg HS, Lewis PJ, Regenold W, Wagner HN Jr. Paralimbic hypoperfusion in unipolar depression. J Nucl Med 1994;35:929-934. 
Supplementary Table 1. Correlations between mean oxy- $\mathrm{Hb}$ changes and demographic or clinical variables

\begin{tabular}{clcc}
\hline \multicolumn{1}{c}{ Correlation analysis } & \multicolumn{1}{c}{ Clinical variables } & Correlation coefficient & p value \\
\hline Pearson & Age & -0.454 to 0.426 & 0.078 to 0.974 \\
Spearman & Estimated IQ & -0.440 to 0.328 & 0.116 to 1.000 \\
& Years of educations & -0.348 to 0.348 & 0.170 to 1.000 \\
& HAM-D score & -0.200 to 0.681 & 0.003 to 0.990 \\
& Illness duration & -0.465 to 0.479 & 0.070 to 0.994 \\
& Duration of depressive episodes & -0.263 to 0.471 & 0.076 to 0.990 \\
& Task performance & -0.473 to 0.183 & 0.048 to 0.988 \\
& Imipramine dose & -0.493 to 0.258 & 0.052 to 0.987 \\
& Chlorpromazine dose & -0.411 to 0.200 & 0.144 to 1.000 \\
& Diazepam dose & -0.321 to 0.263 & 0.225 to 1.000 \\
\hline
\end{tabular}

oxy-Hb: oxygenated hemoglobin, IQ: intelligence quotient, HAM-D: 17-item Hamilton Rating Scale for Depression 
Supplementary Table 2. Correlations between mean oxy-Hb changes and the other five BACS-J subscale scores

\begin{tabular}{clcc}
\hline \multicolumn{1}{c}{ Correlation analysis } & \multicolumn{1}{c}{ Clinical variables } & Correlation coefficient & p value \\
\hline Pearson & Verbal memory & -0.641 to 0.497 & 0.010 to 0.977 \\
& Verbal fluency & -0.612 to 0.552 & 0.009 to 0.985 \\
& Attention and speed of information processing & -0.554 to 0.276 & 0.032 to 0.994 \\
& Executive function & -0.418 to 0.335 & 0.121 to 0.989 \\
Spearman & Working memory & -0.447 to 0.383 & 0.063 to 0.965 \\
\hline
\end{tabular}

oxy-Hb: oxygenated hemoglobin, BACS-J: Japanese version of the Brief Assessment of Cognition in Schizophrenia 